Supplement of Biogeosciences, 12, 5995-6015, 2015

http://www.biogeosciences.net/12/5995/2015/

doi:10.5194/bg-12-5995-2015-supplement

(C) Author(s) 2015. CC Attribution 3.0 License.

(c) (i)

Supplement of

\title{
Interpreting canopy development and physiology using a European phenology camera network at flux sites
}

\section{Wingate et al.}

Correspondence to: L. Wingate (lisa.wingate@bordeaux.inra.fr)

The copyright of individual parts of the supplement might differ from the CC-BY 3.0 licence. 


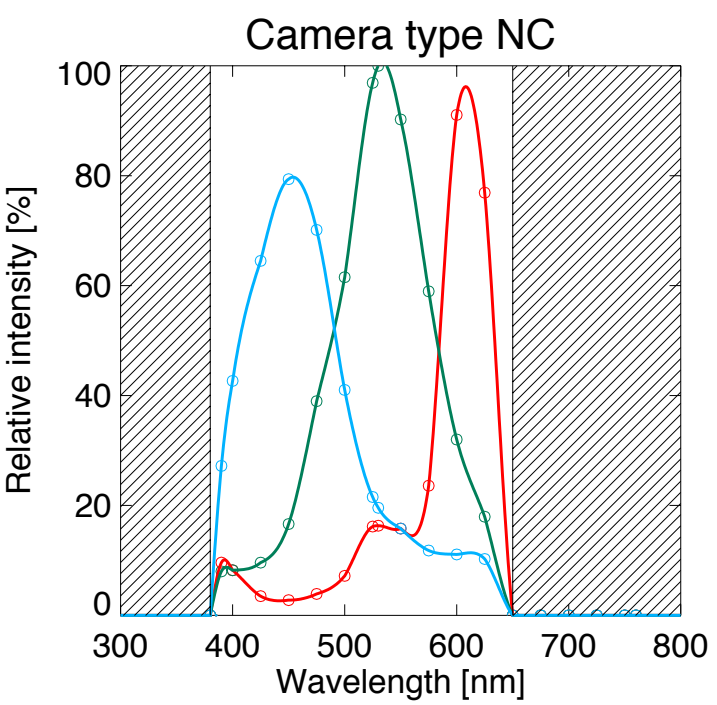

4

5 Figure S1. Spectral RGB responses of the NetCam camera sensors, as well as their IR

6 and UV cut-off filters. To ease the comparison with other cameras and because we are

7 only interested in colour fractions the spectral responses have been expressed relative 8 to the maximum value.

9 


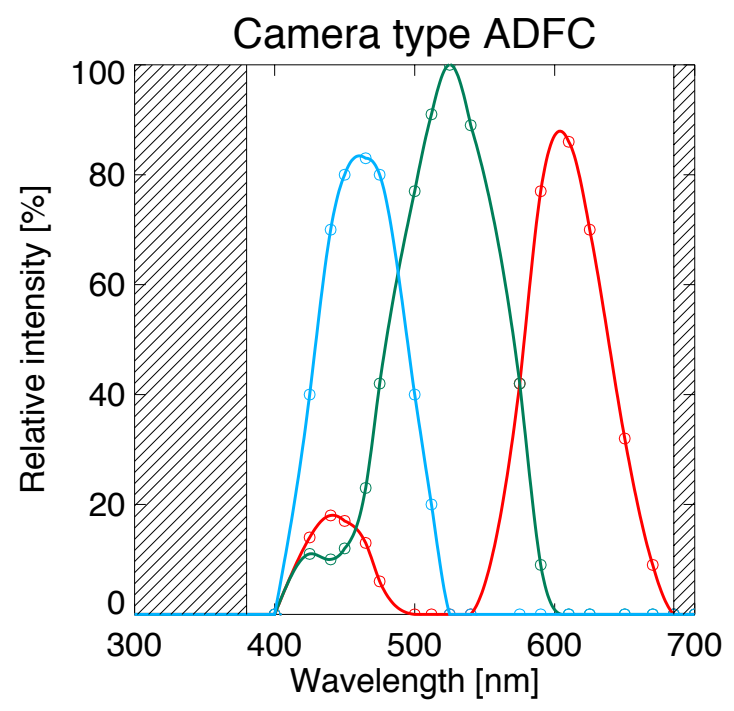

3 Figure S2. Same as Fig. S1 but for the Nikon Coolpix camera (data courtesy of Dr. T.

4 Nakaji). These data where obtained using a monochrometer (Monochrometer PXE-300,

5 Jasco Co., Tokyo, 1996) coupled to a grating unit in order to emit a beam within the

6 VIS-NIR target wavelengths (400 to $1100 \mathrm{~nm}$ with $23 \mathrm{~nm}$ FWHM). The intensity of the

7 output beam was then checked with a spectroradiometer (ASD Field Spec PRO) at the

8 beginning of the camera experiment. Thereafter, images of the diffused beam directed

9 on to a Spectralon card were captured by a range of commercial cameras. Images were

10 captured whilst varying the wavebands (20 bands) from 408 to $782 \mathrm{~nm}$. The experiment

11 was conducted in a dark room, and the geometry and camera settings (white balance,

12 shutter speed, etc.) were adjusted. The DN of image RGB pixels were then corrected

13 for dark noise and the ratio between DN and input energy was calculated and expressed

14 as a relative value to estimate the camera sensitivity differences.

15 


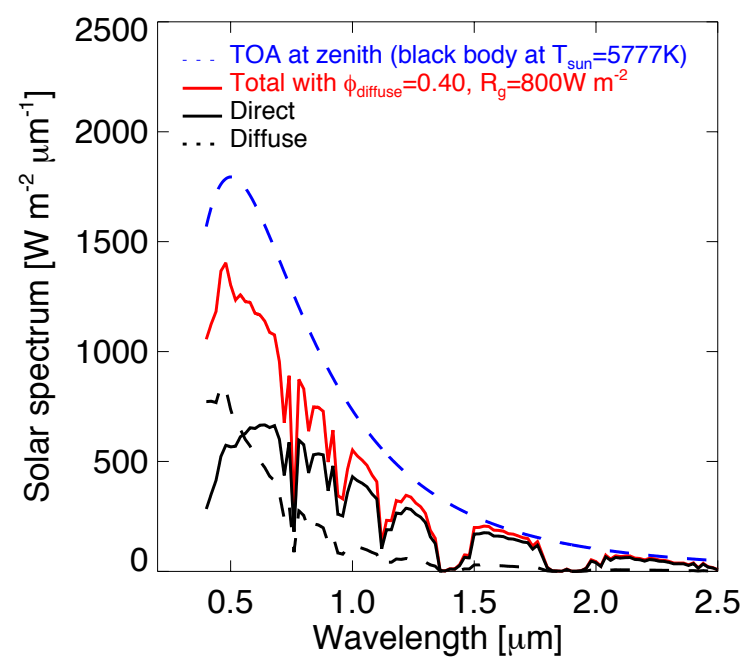

3 Figure S3. Solar spectra for top-of-the-atmosphere (TOA), and top-of-the-canopy 4 (TOC) incoming radiation, assuming $40 \%$ diffuse radiation. The TOA spectra is 5 computed for a black body at $5777 \mathrm{~K}$ while the TOC spectra is computed from pure 6 direct and diffuse spectra calculated using the $6 \mathrm{~S}$ radiative transfer model (François et $7 \quad$ al., 1999) also shown in the figure.

8 


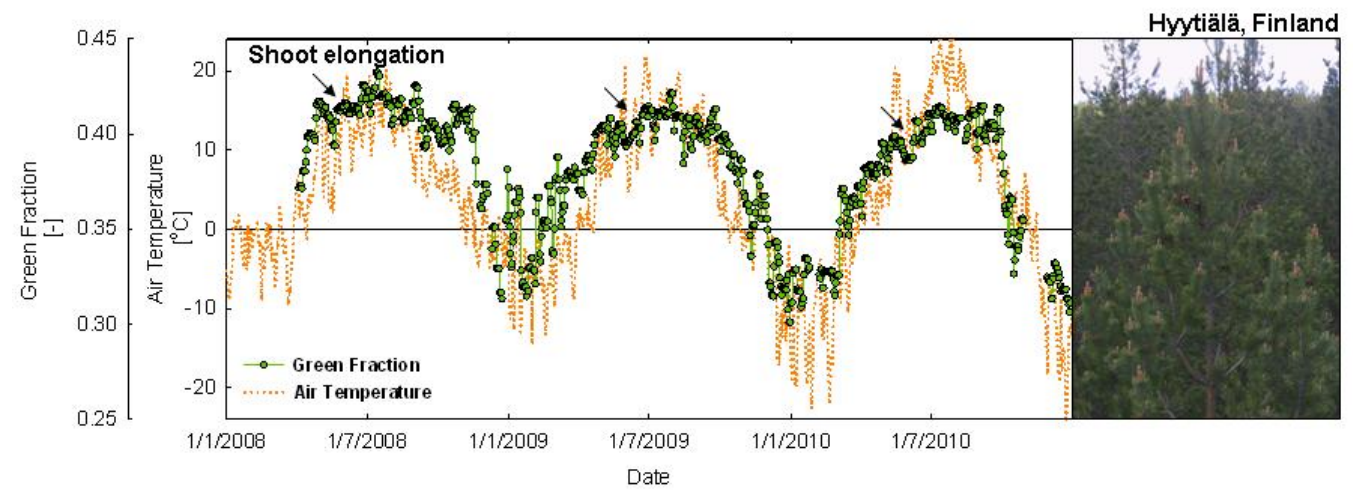

3 Figure S4. Green fraction and daily air temperature time-series at the evergreen

4 conifer forest Hyytiälä. Arrows indicate periods when new shoots elongate and a

5 typical image during this phenological event is also shown for illustration. 


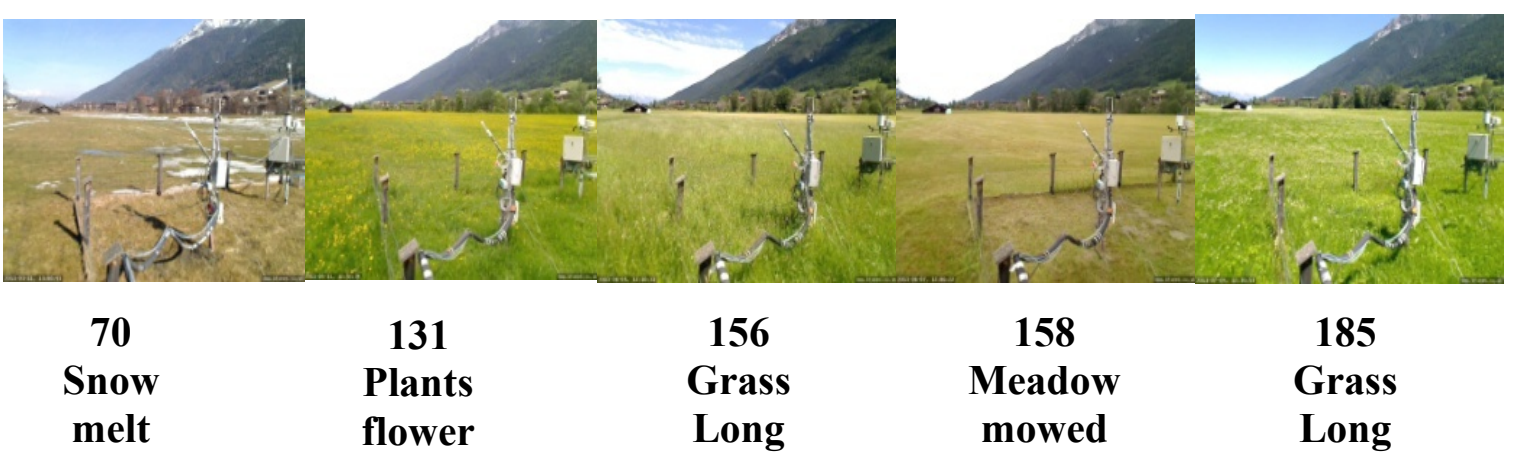

5

6

7 Figure S5. Digital photographs related to Figure 7 illustrating contrasting changes in

8 scene over the growing season at the Neustift flux site located in Innsbruck, Austria 9 during 2009. 


\section{Figure S6}

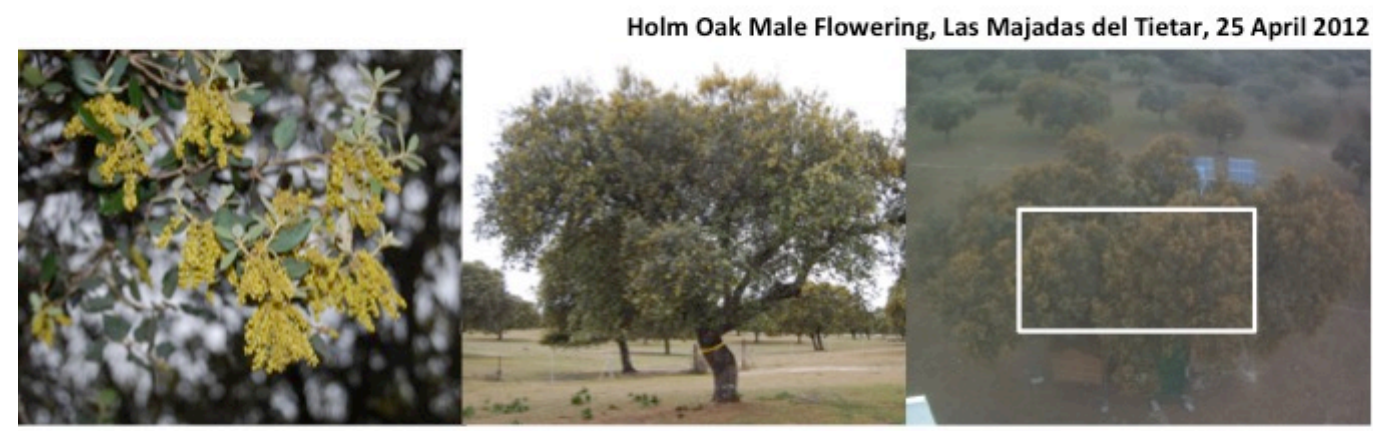

3

4 Figure S6. Digital photographs that illustrate the male flowering at the Las Majadas

5 del Tietar evergreen broadleaf forest during April 2012.

6 
Figure S7

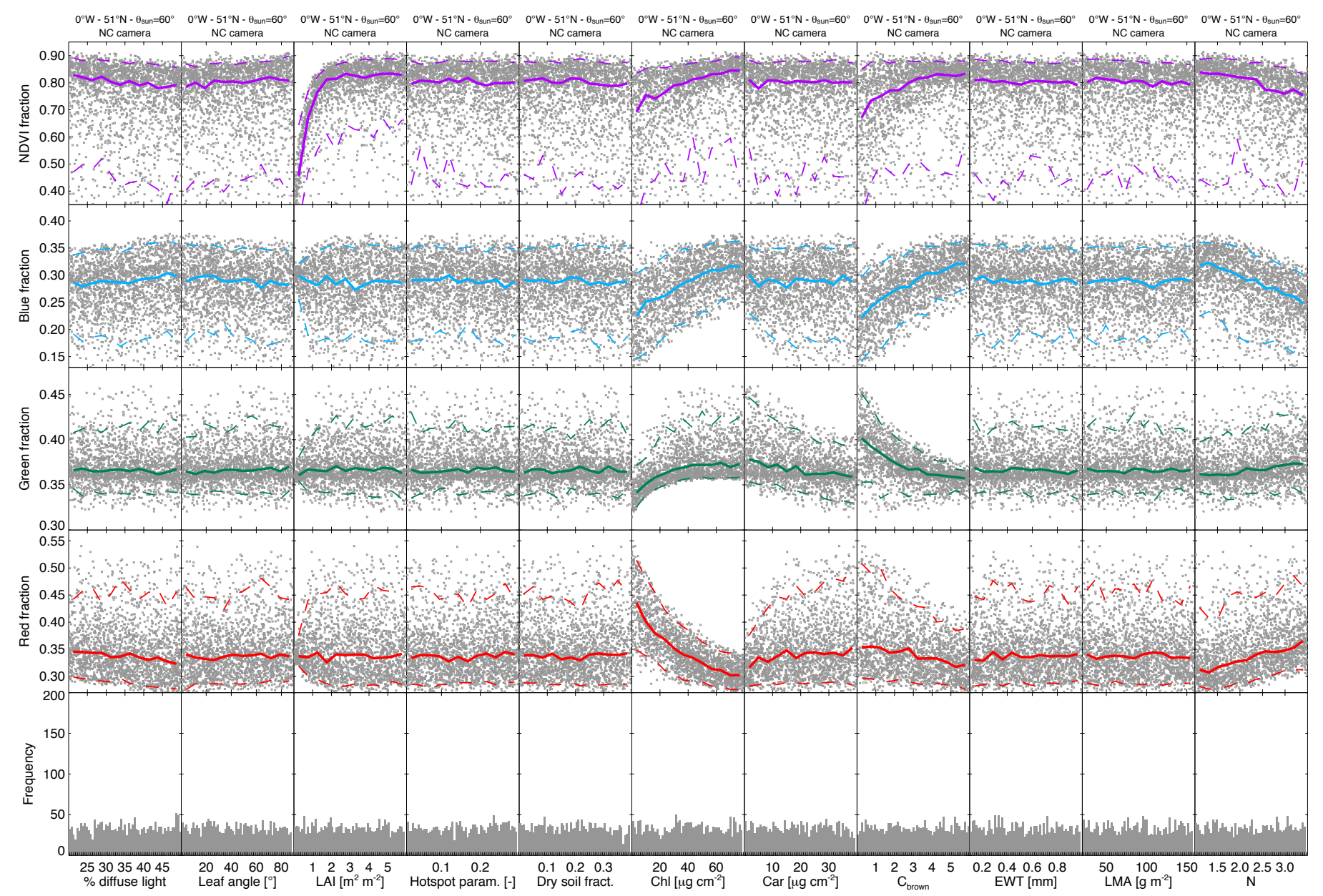


2 Figure S7. Sensitivity of modelled RGB fractions and NDVI for the NetCam camera at the Alice Holt deciduous broadleaf forest site, as 3 predicted by the PROSAIL model and assuming no correlation between model parameters, and a constant solar elevation of $60^{\circ}$. The NDVI is 4 computed using the camera view angle and the same wavebands as for MODIS NDVI (545-565 $\mathrm{nm}$ for red and 841-871 nm for near infrared). 


\section{Figure S8}

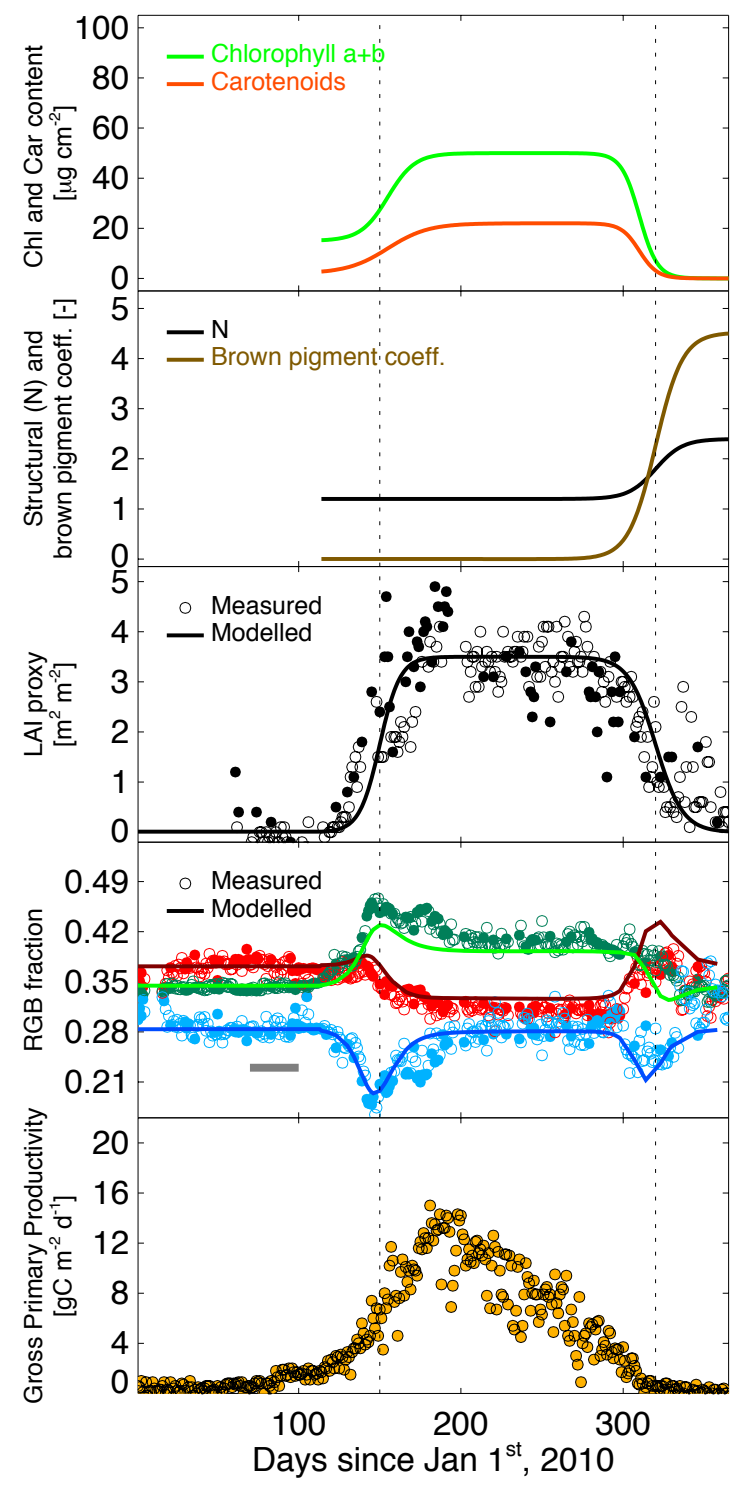

4 Figure S8. As in Fig. 12, but for the Nikon Coolpix camera (i.e. using the camera

5 spectral response from Fig. S2, rather than from Fig. S1). The IDL code and data 6 required to generate these figures can be downloaded from the repository 7 (https://bitbucket.org/jerome_ogee/webcam_network_paper).

8 